OPEN ACCESS

Edited by:

Zaleha Abdullah Mahdy,

National University of

Malaysia, Malaysia

Reviewed by:

Nik Hazlina Nik Hussain,

Universiti Sains Malaysia

(USM), Malaysia

Bavanandam Naidu,

Ministry of Health, Malaysia

*Correspondence:

Tsung-Hsien Lee

jackth.lee@gmail.com

James Cheng-Chung Wei

wei3228@gmail.com

Specialty section:

This article was submitted to

Obstetrics and Gynecology,

a section of the journal

Frontiers in Medicine

Received: 20 June 2020

Accepted: 18 August 2020

Published: 23 September 2020

Citation:

Wu C-H, Chiu L-T, Chang Y-J,

Lee C-I, Lee M-S, Lee T-H and Wei JC-C (2020) Hypertension Risk in Young Women With Polycystic Ovary

Syndrome: A Nationwide

Population-Based Cohort Study.

Front. Med. 7:574651.

doi: 10.3389/fmed.2020.574651

\section{Hypertension Risk in Young Women With Polycystic Ovary Syndrome: A Nationwide Population-Based Cohort Study}

\author{
Cheng-Hsuan Wu $u^{1,2,3}$, Lu-Ting Chiu ${ }^{4}$, Yu-Jun Chang ${ }^{5}$, Chun-I Lee ${ }^{1,6,7}$, Maw-Sheng Lee ${ }^{1,6,7}$, \\ Tsung-Hsien Lee ${ }^{1,6,7 *}$ and James Cheng-Chung Wei ${ }^{1,8,9 *}$ \\ ${ }^{1}$ Institute of Medicine, Chung Shan Medical University, Taichung, Taiwan, ${ }^{2}$ Women's Health Research Laboratory, Changhua \\ Christian Hospital, Changhua, Taiwan, ${ }^{3}$ School of Medicine, Kaohsiung Medical University, Kaohsiung, Taiwan, \\ ${ }^{4}$ Management Office for Health Data, Clinical Trial Research Center, China Medical University Hospital, Taichung, Taiwan, \\ ${ }^{5}$ Epidemiology and Biostatistics Center, Changhua Christian Hospital, Changhua, Taiwan, ${ }^{6}$ Department of Obstetrics and \\ Gynecology, Chung Shan Medical University Hospital, Taichung, Taiwan, ${ }^{7}$ Division of Infertility, Lee Women's Hospital, \\ Taichung, Taiwan, ${ }^{8}$ Department of Allergy, Immunology and Rheumatology, Chung Shan Medical University Hospital, \\ Taichung, Taiwan, ${ }^{9}$ Graduate Institute of Integrated Medicine, China Medical University, Taichung, Taiwan
}

Objective: A number of publications have assessed the prevalence of hypertension in polycystic ovary syndrome (PCOS) patients with inconclusive results. Since in general populations the occurrence of hypertension is related to age and comorbidities, we investigated the incidence rate and hazard ratios (HRs) of hypertension between healthy subjects and young women with PCOS as well as comorbidities.

Methods: We conducted a population-based retrospective cohort study by using the National Health Insurance Research Database in Taiwan. The cohort included women with the diagnosis of PCOS between 2000 and 2012. Those without PCOS were selected as the control group at a ratio of 4:1 by an age-matched systematic random-sampling method. Cox proportional hazard regression analysis was used to determine the effects of PCOS on the risks of developing hypertension. Stratification analysis was performed to elucidate the interaction among PCOS and the comorbidities, which affect the incidence of hypertension.

Results: The PCOS cohort consisted of 20,652 patients with PCOS and the comparison cohort consisted of 82,608 matched patients without PCOS. There was no difference in the distribution of age between the PCOS cohort and the comparison cohort $(29.1 \pm 6.8$ vs. $29.0 \pm 6.5, p=0.32$ ). The incidence rates of hypertension were 7.85 and 4.23 per 1,000 person-years in the PCOS and comparison groups, respectively. A statistically significant higher risk of hypertension was found in the PCOS cohort (adjusted $\mathrm{HR}=1.62$, $95 \%$ confidence interval $=1.48-1.76$ ) than in the comparison cohort. After a joint analysis of comorbidities, the adjusted HR of hypertension was 9.44 (95\% confidence interval $=7.27-12.24$ ) for PCOS patients with comorbidities of diabetes mellitus (DM) and hyperlipidemia compared with women with neither PCOS nor DM and hyperlipidemia. 
Conclusion: The risk of developing hypertension in young women with PCOS was higher than in controls in this cohort study. The comorbidities of DM and hyperlipidemia could interact with PCOS patients and further increase the risk of hypertension. An earlier screening for hypertension and comorbidities in patients with PCOS may be warranted, even in young women.

Keywords: hypertension, polycystic ovarian syndrome, population-based cohort study, young women, comorbidities

\section{INTRODUCTION}

Polycystic ovary syndrome (PCOS) is characterized by ovulatory dysfunction, hyperandrogenism, and polycystic ovarian morphology when other causes are excluded according to the Rotterdam criteria (1). PCOS is the most common endocrine disease affecting the women of reproductive age. Insulin resistance (IR) and androgen excess are prevalent in patients with PCOS $(2,3)$. Previous studies have demonstrated that almost all cardiovascular risk factors including IR, diabetes mellitus (DM), dyslipidemia, obesity, hypertension, and metabolic syndrome are associated with PCOS (4-8). These risk factors are present even in young patients with PCOS and predispose to the development of endothelial dysfunction, early atherosclerosis and cardiovascular disease (CVD) (9-12).

Patients with PCOS, especially with hyperandrogenic phenotype, are exposed to several cardiometabolic risk factors that increase their chance for developing hypertension (13). Androgen excess in PCOS may also directly affect the vascular properties of arterial walls involved in the atherogenic process (14). Although hypertension represents one of the main cardiovascular risk factors in general populations, previous studies have shown inconsistent results on hypertension in PCOS. Recently, a meta-analysis confirmed a greater risk of developing hypertension in patients with PCOS but demonstrated that this risk is increased only in the women of reproductive age with PCOS (15). However, another systemic review and meta-analysis showed that no significant difference for hypertension between non-obese women with PCOS and controls (16).

$\mathrm{DM}$, dyslipidemia, asthma, chronic kidney disease (CKD), and CVD are also some comorbidities that are prevalent in the patients with hypertension (17-21). In addition, given the multitude of comorbidities associated with PCOS, such as DM (16), dyslipidemia (22), CKD (23), asthma (24), chronic obstructive pulmonary disease (COPD) (25), and $\operatorname{CVD}(9,11)$, the objective of this study is to investigate the risk of hypertension associated with the women of reproductive age with PCOS and the abovementioned comorbidities in a population-based cohort database.

\section{MATERIALS AND METHODS}

\section{Data Source}

This is a retrospective population-based cohort study. The Taiwan government launched the National Health Insurance program in 1995, which contains more than 99\% Taiwanese residents through a universal single-payer health system. In this study, we used the data of the Longitudinal Health Insurance Database 2000 (LHID 2000), which consists of registration files and original claims data from 1996 to 2013 of 1 million patients randomly selected from the National Health Insurance Research Database (NHIRD). Meanwhile, many studies have provided validation to the database $(9,26,27)$. As all personal information is encrypted with de-identification process for research purposes in NHIRD database, no patient's consent was required. The LHID identifies diseases were based on the International Classification of Diseases, Ninth Revision, Clinical Modification (ICD-9-CM). This study was approved by the Research Ethics Committee of China Medical University and Hospital in Taiwan (CMUH-104REC2-115-CR-4).

\section{Study Population}

Patients who were diagnosed with PCOS (ICD-9-CM 256.4) were recruited in this cohort study between January 1,2000, and December 31, 2012. We excluded patients who were diagnosed with PCOS based on A code: A189, not ICD-9-CM code: 256.4 between January 1, 1996, and December 31, 1999. We defined the diagnosis date of PCOS as the index date. The exclusion criteria included the followings: (1). age $<18$ years old, (2). hypertension history, (3). PCOS index year $<2000$. Female patients without PCOS during the study period were randomly selected from the same database for the comparison cohort. The comparison cohort was frequency-matched with the PCOS cohort at a 4:1 ratio according to age (every 5 years) and the index year. The index date for the comparison cohort was randomly assigned. Figure 1 illustrates the selection procedure.

The event of interest in the study was a new diagnosis of hypertension (ICD-9-CM codes 401-405) with at least two outpatient visits or at least one hospitalization record from January 1, 2000 to December 31, 2013. Each patient was monitored from the index year until their diagnosis with hypertension; withdrawal from insurance; or at the end of December 31, 2013, whichever came first.

Comorbidities that could be potentially associated with PCOS and hypertension were included as follows: diabetes mellitus (DM; ICD-9-CM code 250), hyperlipidemia (ICD-9-CM code 272), chronic obstructive pulmonary disease (COPD; ICD-9CM code 490-492, 494 and 496), asthma (ICD-9-CM code 493), chronic kidney disease (CKD; ICD-9-CM 250.4, 274.1, 283.11, 403, 404, 440.1, 442.1, 447.3, 572.4, 580-588, 642.1, and 646.2), coronary artery disease (CAD; ICD-9-CM code 410-414), and 


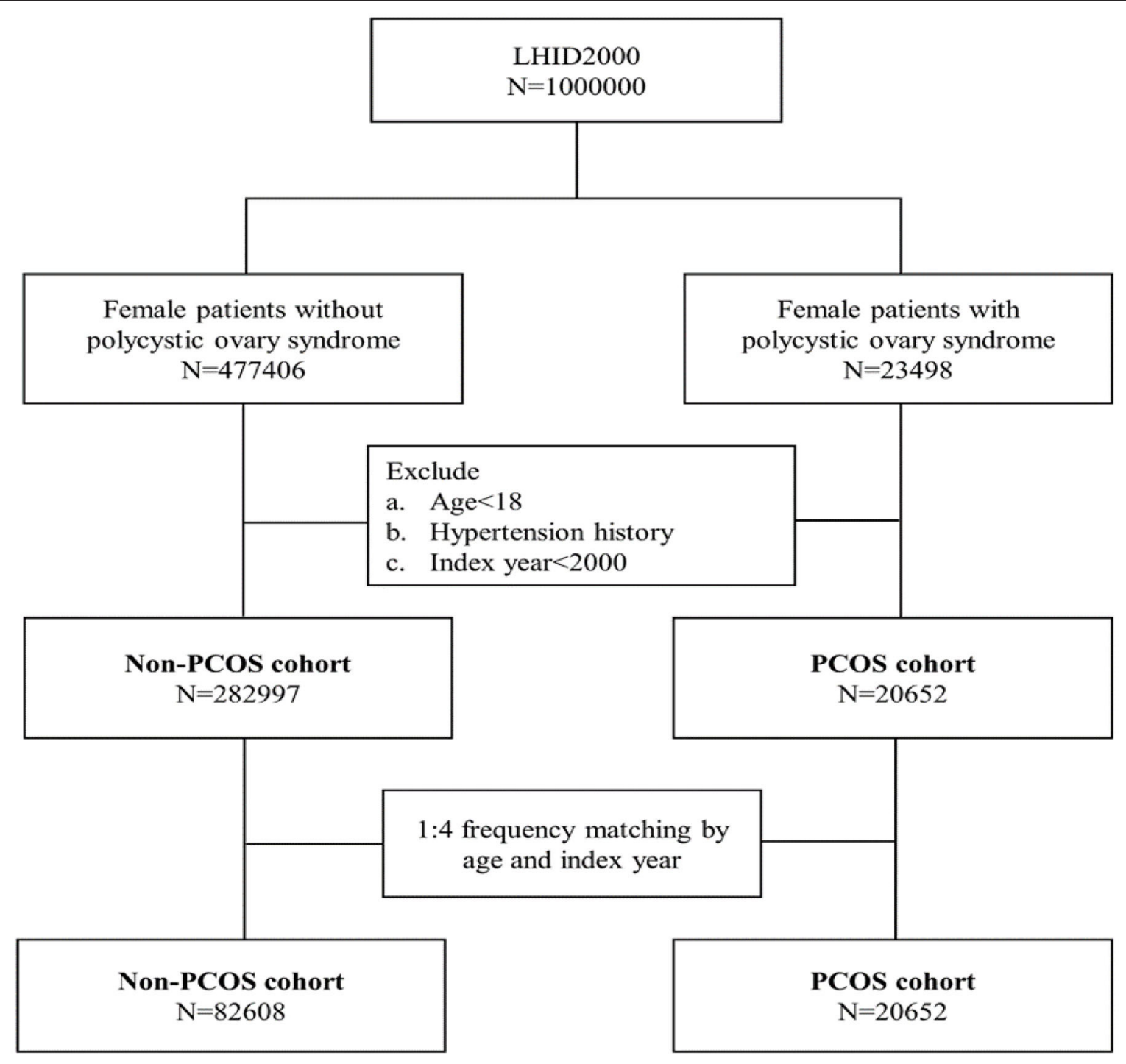

FIGURE 1 | Flow chart of the subject selection process.

congestive heart failure (CHF; ICD-9-CM code 398.91, 402.01, 402.11, 402.91, 404.01, 404.03, 404.11, 404.13, 404.91, 404.91, 404.93, and 428).

\section{Statistical Analysis}

We described the age and follow-up time by mean with standard deviation and tested the difference between the PCOS and comparison cohorts by using the $t$-test. The categorical variables were presented as the numbers with percentage and tested the difference by using the Chi-square test. The hazard ratio (HR) with 95\% confidence interval (CI) for hypertension was estimated by Cox proportional regression analysis, which was adjusted for the potential confounding variables, such as age and comorbidities between the PCOS cohort and comparison cohorts. Stratification analysis was performed to elucidate the interaction among PCOS and the comorbidities, which affect the incidence of hypertension. To further clarify the correlation between PCOS with comorbidities and hypertension, we subsequently conducted a joint analysis of DM, hyperlipidemia, and PCOS, and compared the risk of hypertension in each combination. In addition, we measured and compared the cumulative incidence curves for hypertension between the PCOS cohort and tested the difference of the curves by using the log rank test. All statistical analyses were performed by using SAS version 9.4 (Version 9.4, SAS Institute Inc., Cary, NC, USA). The significance level for statistical analysis was set at $P<0.05$.

\section{RESULTS}

The PCOS cohort consisted of 20,652 patients with PCOS and the comparison cohort consisted of 82,608 matched patients without 
PCOS (Table 1). There was no difference in the distribution of age between the PCOS cohort and the comparison cohort $(29.0 \pm 6.5$ vs. $29.1 \pm 6.8, p=0.32)$. Patients with PCOS

TABLE 1 | Baseline characteristics of patients with and without polycystic ovarian syndrome.

\begin{tabular}{lccc}
\hline & \multicolumn{2}{c}{ Polycystic ovarian syndrome } & \multirow{2}{*}{$\boldsymbol{p}$-value } \\
\cline { 2 - 3 } & No $(\boldsymbol{n}=\mathbf{8 2 , 6 0 8 )}$ & Yes $(\boldsymbol{n}=\mathbf{2 0 , 6 5 2})$ & \\
\hline Age (years), $\boldsymbol{n}$ (\%) & & & \multirow{2}{*}{1.00} \\
$\leq 30$ & $48,656(58.9)$ & $12,164(58.9)$ & \\
$>30$ & $33,952(41.1)$ & $8,488(41.1)$ & \\
Mean (SD) & $29.1(6.8)$ & $29.0(6.5)$ & 0.32 \\
Follow-up time (years) & & & \\
Mean (SD) & $4.9(3.5)$ & $4.8(3.5)$ & 0.19 \\
Comorbidity, $\boldsymbol{n}$ (\%) & & & \\
Diabetes mellitus & $1,218(1.5)$ & $1,048(5.1)$ & $<0.0001$ \\
Hyperlipidemia & $1,858(2.3)$ & $1,126(5.5)$ & $<0.0001$ \\
COPD & $10,092(12.2)$ & $3,302(16.0)$ & $<0.0001$ \\
Asthma & $4,440(5.4)$ & $1,480(7.2)$ & $<0.0001$ \\
Chronic kidney disease & $975(1.2)$ & $363(1.8)$ & $<0.0001$ \\
Coronary artery disease & $781(1.0)$ & $118(0.6)$ & $<0.0001$ \\
Congestive heart failure & $120(0.2)$ & $20(0.1)$ & 0.19 \\
\hline
\end{tabular}

COPD, chronic obstructive pulmonary disease; SD, standard deviation. tended to have a higher proportion of DM, hyperlipidemia, COPD, asthma, CKD, and CAD than those in the control cohort $(p<0.0001)$. The mean follow-up periods were similar in the two cohorts $(4.9 \pm 3.5$ years vs. $4.8 \pm 3.5$ years, $p=0.19)$.

The results of the Kaplan-Meier analysis showed that the PCOS cohort had a significantly higher cumulative incidence of hypertension than the comparison cohort (logrank test, $p<0.001$ ) at the end of follow-up (Figure 2). The overall incidence rates of hypertension in the PCOS cohort and the comparison cohort were 7.85 and 4.23 per 1,000 person-years, respectively. After adjusting for age and all comorbidities, the risk of hypertension was significantly higher in the patients with PCOS than in the comparison cohort by an adjusted $\mathrm{HR}$ of 1.62 (95\% CI $=1.48-$ 1.76, $p<0.001)$. The incidence of hypertension increases with age. Compared with patients aged $\leq 30$ years, the risk of hypertension occurrence was 2.88-fold higher in patients aged above 30 years (95\% CI $=2.65-3.13, p<$ 0.001). As for potential comorbidities, patients with DM (adjusted HR $=3.00 ; 95 \% \mathrm{CI}=2.61-3.45$ ), hyperlipidemia (adjusted HR $=2.42 ; 95 \% \mathrm{CI}=2.11-2.79$ ), asthma (adjusted $\mathrm{HR}=1.27 ; 95 \% \mathrm{CI}=1.04-1.56$ ), $\mathrm{CKD}$ (adjusted $\mathrm{HR}=1.53$; $95 \% \mathrm{CI}=1.20-1.95$ ), and $\mathrm{CAD}$ (adjusted $\mathrm{HR}=1.82$; 95\% CI $=1.42-2.33$ ) are at a relatively higher risk of developing hypertension than patients without comorbidities (Table 2).

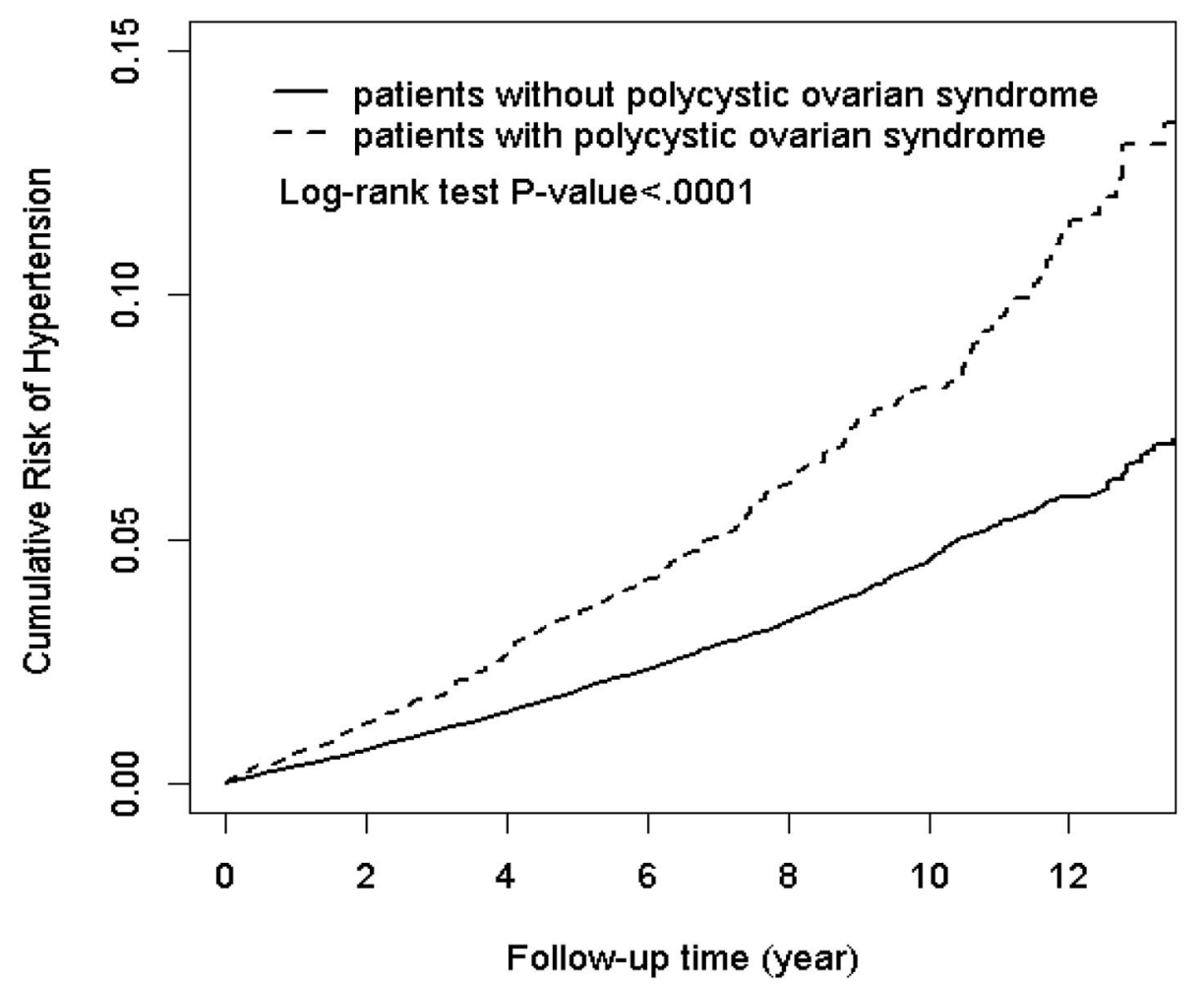

FIGURE 2 | Kaplan-Meier plot of the cumulative risk of hypertension in patients with and without polycystic ovarian syndrome (log-rank $<0.0001)$. 
TABLE 2 | The incidence and HRs for risk of hypertension associated with PCOS, age and comorbidities.

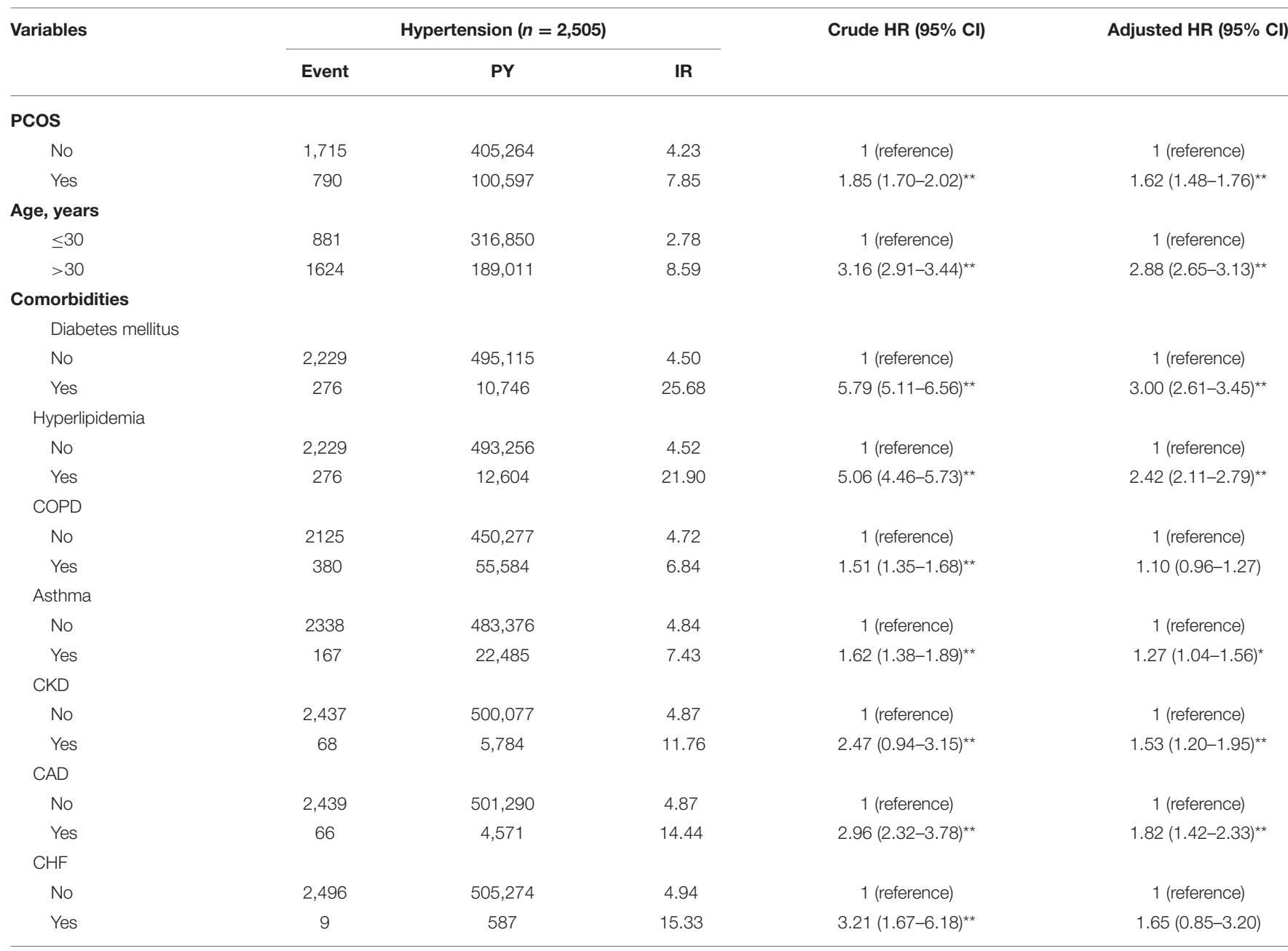

${ }^{*} p<0.05,{ }^{* *} p<0.001$.

HR adjusted for age, diabetes mellitus, hyperlipidemia, COPD, asthma, CKD, CAD and CHF.

$C A D$, coronary artery disease; $C H F$, congestive heart failure; $\mathrm{Cl}$, confidence interval; $C K D$, chronic kidney disease; COPD, chronic obstructive pulmonary disease; HR, hazard ratio; IR, incidence rate, per 1,000 person-years; PCOS, polycystic ovarian syndrome; PY, person-years.

The HRs of hypertension in PCOS and control group by stratification analysis with various comorbidities were demonstrated in Table 3. It is interesting to note that the risk of hypertension in patients with PCOS is more prominent at young age ( $\leq 30$ years, adjusted HR: $2.20,95 \% \mathrm{CI}=1.91-2.54$, $p<0.001$; whereas for patients older than 30 years, adjusted HR: $1.30,95 \% \mathrm{CI}=1.17-1.46, p<0.001)$. When stratified by comorbidity, several significantly increased risks of hypertension were found in the following group of patients with PCOS: patients with and without DM, hyperlipidemia, COPD, and asthma. Nevertheless, CKD, CAD, and CHF did not change the HR for the incidence of hypertension in the patients with PCOS (Table 3).

Among the comorbidities, DM alone was associated with hypertension development with an adjusted HR of 3.19 (95\% CI $=2.52-4.04, p<0.001$ ) (Table 4). The adjusted HR of hypertension increased to 6.62 for PCOS patients with DM compared with women with neither PCOS nor comorbidities of DM and hyperlipidemia. The corresponding adjusted HR of hypertension even increased to $9.44(95 \% \mathrm{CI}=7.27-12.24, p<$ 0.001 ) for PCOS patients with DM and hyperlipidemia (Table 4).

\section{DISCUSSION}

We conducted this population-based cohort study based on a large size of young female population with PCOS at an average age of 29 years. Young women are generally at a low risk of hypertension. Nevertheless, this study confirms that the risk of developing hypertension in young female population with PCOS is $62 \%$ higher than those without PCOS (adjusted HR $=1.62$, $95 \% \mathrm{CI}=1.48-1.76)$. Meanwhile, the comorbidities of DM and hyperlipidemia were also related with the risk of hypertension and could interact with women having PCOS, further increasing the risk of hypertension.

Previous studies on the relations between PCOS and hypertension remain debatable. Recently, a cross-sectional study 
TABLE 3 | The incidence and HRs for risk of hypertension in patients with and without PCOS stratified by age and comorbidities.

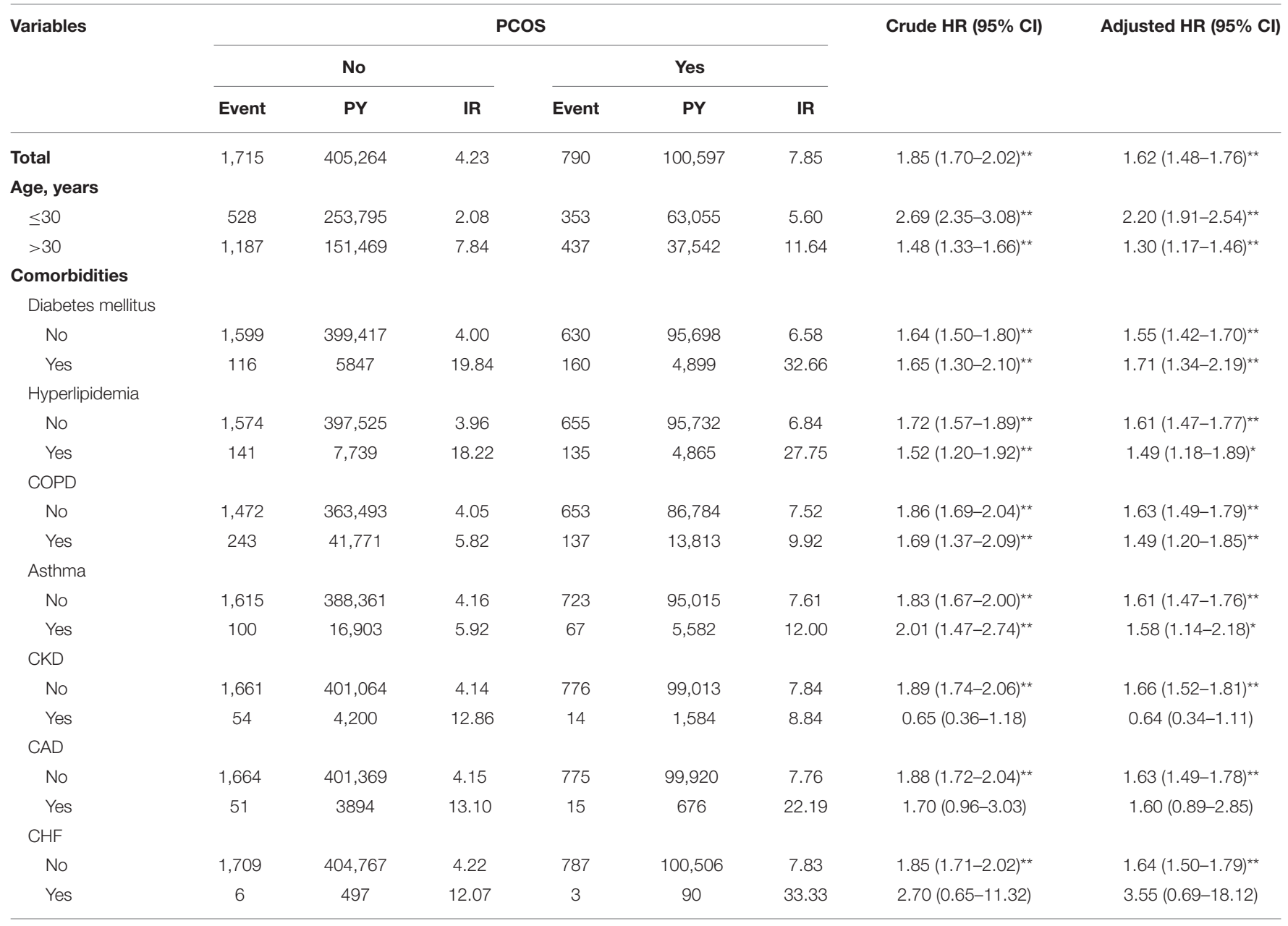

${ }^{*} p<0.01,{ }^{* *} p<0.001$.

HR adjusted for age, diabetes mellitus, hyperlipidemia, COPD, asthma, CKD, CAD and CHF.

$C A D$, coronary artery disease; $C H F$, congestive heart failure; $\mathrm{Cl}$, confidence interval; $C K D$, chronic kidney disease; COPD, chronic obstructive pulmonary disease; HR, hazard ratio; IR, incidence rate, per 1,000 person-years; PCOS, polycystic ovarian syndrome; PY, person-years.

TABLE 4 | The incidence and HRs for risk of hypertension associated with PCOS, diabetes and hyperlipidemia.

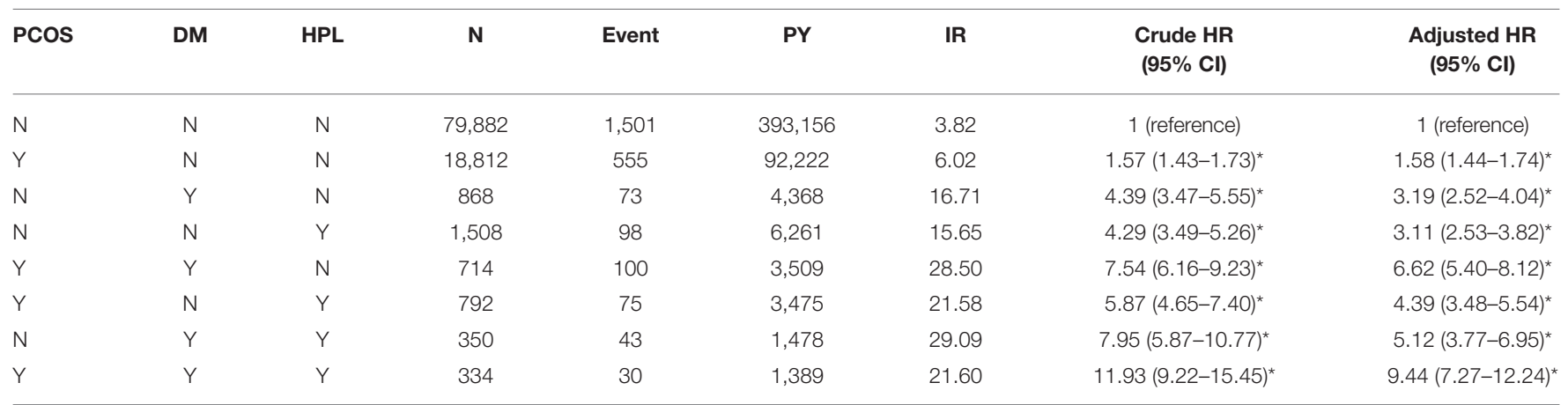

${ }^{*} p<0.001$.

HR adjusted for age, chronic obstructive pulmonary disease, asthma, chronic kidney disease, coronary artery disease, and congestive heart failure.

Cl, confidence interval; DM, diabetes mellitus; HPL, hyperlipidemia; HR, hazard ratio; IR, incidence rate, per 1,000 person-years; PCOS, polycystic ovarian syndrome; PY, person-years. 
in Brazil found the prevalence of hypertension at $65 \%$ among young patients with PCOS (mean age: $25-26.5$ years) vs. $41 \%$ among control women without PCOS (mean age: 29 years) (28). A meta-analysis study revealed a greater risk of hypertension in women with PCOS but demonstrated that this pooled relative risk is only increased in the women of reproductive age with PCOS (1.70-fold, 95\% CI: 1.43-2.07) (15). The abovementioned results are consistent with our findings. We found an adjusted HR of 1.62 for developing hypertension after controlling for age and other comorbidities. However, another systemic review observed no significant difference for hypertension between nonobese women of reproductive age with and without PCOS (16). A possible explanation for this discrepancy is that body mass index (BMI) was not included for analysis in our study. Obesity is considered as a key factor for the alteration of blood pressure in women with PCOS $(29,30)$.

The mechanism underlying the increased prevalence of hypertension in PCOS has been linked to several factors such as IR and hyperinsulinemia (31), hyperandrogenism (8), obesity (30), and heart autonomic dysfunction (32). IR interferes with the endothelium-dependent vasodilatation mechanisms causing vascular muscle wall hypertrophy and compensatory hyperinsulinemia further contributes to the development of hyperandrogenemia (33). The hyperandrogenic state of PCOS develops exacerbated cardio-metabolic profile with consequent endothelial dysfunction and elevated blood pressure (34). Additionally, IR-related compensatory hyperinsulinemia may affect blood pressure through an imbalance of autonomic nervous system, decreased production of nitric oxide, and an increased reabsorption of renal sodium (28).

Our results found that patients with PCOS and patients with hypertension share similar characteristics of chronic comorbidities $(35,36)$. Patients with PCOS tended to have a higher proportion of DM, hyperlipidemia, COPD, asthma, CKD, and CAD than those in the control cohort $(p<0.0001)$ (Table 1 ). Meanwhile, patients with DM, hyperlipidemia, asthma, or CKD had a relatively higher risk of developing hypertension than patients without comorbidities (Table 2). DM or hyperlipidemia alone was a stronger risk factor than PCOS alone in association with the development of hypertension (adjusted HR: 3.00, 2.42, and 1.62, respectively) (Table 2). After stratification analysis, women with hyperlipidemia, COPD, asthma, CKD, or CAD did not further modify the hypertension risk in PCOS patients than in controls. By contrast, women with DM could further increase the risk of hypertension in patients with PCOS (Table 3).

Age is another substantial risk factor for the manifestation of hypertension. In this population-based analysis, the risk of hypertension occurrence was 2.88 -fold $(95 \% \mathrm{CI}=2.65-3.13$, $p<$ 0.001 ) higher in patients aged above 30 years as compared with the patients aged $\leq 30$ years (Table 2 ). After stratification analysis, it is interesting to find that an early diagnosis of PCOS at a younger age ( $\leq 30$ years) increased the incidence of hypertension (Table 3). This finding is the same as the one demonstrated by the log-rank test. Therefore, earlier screening for hypertension in patients with PCOS may be warranted, even in young women.

This study highlighted the risk of developing hypertension in the women of reproductive age with PCOS comorbid with DM and hyperlipidemia by an adjusted HR of 9.44 (95\% CI $=7.27$ 12.24, $p<0.001$ ) (Table 4). Therefore, once the diagnosis of PCOS is made, there should be an assessment of metabolic status and comorbidities (37). Recently, a systemic study summarized the findings of 16 studies and found a greater risk of CVD in the women of reproductive age with PCOS (5). According to the recently published international PCOS guidelines, the global CVD risk should be routinely assessed in women with PCOS (38). There should be an annual monitoring of glucose, lipid profiles, blood pressure, and weight in these women. All providers engaged in the multidimensional care of women with PCOS should be alarmed of these long-term health risks to provide appropriate screening, counseling, and management options (12).

This retrospective study was performed based on the database NHIRD, which contained all the medical record information of one million Taiwanese randomly selected from the 23 million Taiwan population. All the incomplete data in the medical records for medical cost claim are audited by the authority National Health Insurance Administration. Several limitations to our study should be considered. First, the NHIRD does not provide baseline information on patients, such as BMI, smoking, alcohol consumption, and family history. These are all the risk factors for the development of hypertension. Thus, we were unable to control for these potentially confounding factors. Second, parity is also not recorded in the database. A recent study has reported that nulliparity is associated with a higher risk of pregnancy-associated hypertension (39). Third, we did not compare the different PCOS phenotypes, which may have potentially affected our results. Fourth, this study includes the retrospective design and prospective cohort studies based on the homogeneous populations of large sample size are still needed to verify our results.

\section{CONCLUSION}

In conclusion, our nationwide population-based cohort study provided evidence of an increased risk of hypertension in the women of reproductive age with PCOS. The early diagnosis of PCOS of younger age corresponds to the higher cumulative incidence of hypertension. The comorbidities with DM and hyperlipidemia further increase the risk of developing hypertension. An earlier screening for hypertension and comorbidities in patients with PCOS may be warranted, even in young women.

\section{DATA AVAILABILITY STATEMENT}

All datasets generated for this study are included in the article/supplementary material.

\section{ETHICS STATEMENT}

The studies involving human participants were reviewed and approved by Research Ethics Committee of China Medical University and Hospital in Taiwan (CMUH104-REC2-115-CR-4). Written informed consent 
for participation was not required for this study in accordance with the national legislation and the institutional requirements.

\section{AUTHOR CONTRIBUTIONS}

C-HW and T-HL: conceptualization. T-HL, C-IL, and M-SL: methodology. T-HL and M-SL: validation. L-TC and Y-JC: formal analysis. C-IL: resources. C-HW: writing-original draft preparation. T-HL and JW: writing-review and editing. JW: funding acquisition. All authors have read and agreed to the published version of the manuscript.

\section{REFERENCES}

1. Rotterdam ES. Revised 2003 consensus on diagnostic criteria and longterm health risks related to polycystic ovary syndrome. Fertil Steril. (2004) 81:19-25. doi: 10.1016/j.fertnstert.2003.10.004

2. Azziz R, Carmina E, Chen Z, Dunaif A, Laven JS, Legro RS, et al. Polycystic ovary syndrome. Nat Rev Dis Primers. (2016) 2:16057. doi: $10.1038 /$ nrdp. 2016.57

3. Cooney LG, Dokras A. Beyond fertility: polycystic ovary syndrome and long-term health. Fertil Steril. (2018) 110:794809. doi: 10.1016/j.fertnstert.2018.08.021

4. Chang AY, Oshiro J, Ayers C, Auchus RJ. Influence of race/ethnicity on cardiovascular risk factors in polycystic ovary syndrome, the Dallas Heart Study. Clin Endocrinol. (2016) 85:92-9. doi: 10.1111/cen.12986

5. Ramezani Tehrani F, Amiri M, Behboudi-Gandevani S, BidhendiYarandi R, Carmina E. Cardiovascular events among reproductive and menopausal age women with polycystic ovary syndrome: a systematic review and meta-analysis. Gynecol Endocrinol. (2020) 36:12-23. doi: 10.1080/09513590.2019.1650337

6. Behboudi-Gandevani S, Ramezani Tehrani F, Hosseinpanah F, Khalili D, Cheraghi L, Kazemijaliseh H, et al. Cardiometabolic risks in polycystic ovary syndrome: long-term population-based follow-up study. Fertil Steril. (2018) 110:1377-86. doi: 10.1016/j.fertnstert.2018.08.046

7. Meun C, Gunning MN, Louwers YV, Peters H, Roos-Hesselink J, Roeters van Lennep J, et al. The cardiovascular risk profile of middle-aged women with polycystic ovary syndrome. Clin Endocrinol. (2020) 92:1508. doi: 10.1111/cen.14117

8. Pinola P, Puukka K, Piltonen TT, Puurunen J, Vanky E, Sundstrom-Poromaa I, et al. Normo- and hyperandrogenic women with polycystic ovary syndrome exhibit an adverse metabolic profile through life. Fertil Steril. (2017) 107:78895.e2. doi: 10.1016/j.fertnstert.2016.12.017

9. Ding DC, Tsai IJ, Wang JH, Lin SZ, Sung FC. Coronary artery disease risk in young women with polycystic ovary syndrome. Oncotarget. (2018) 9:8756-64. doi: 10.18632/oncotarget.23985

10. Tarkun I, Arslan BC, Canturk Z, Turemen E, Sahin T, Duman C. Endothelial dysfunction in young women with polycystic ovary syndrome: relationship with insulin resistance and low-grade chronic inflammation. J Clin Endocrinol Metab. (2004) 89:5592-6. doi: 10.1210/jc.2004-0751

11. Glintborg D, Rubin KH, Nybo M, Abrahamsen B, Andersen $M$. Cardiovascular disease in a nationwide population of Danish women with polycystic ovary syndrome. Cardiovasc Diabetol. (2018) 17:37. doi: 10.1186/s12933-018-0680-5

12. Osibogun O, Ogunmoroti O, Michos ED. Polycystic ovary syndrome and cardiometabolic risk: opportunities for cardiovascular disease prevention. Trends Cardiovasc Med. (in press). doi: 10.1016/j.tcm.2019.08.010

13. Joham AE, Boyle JA, Zoungas S, Teede HJ. Hypertension in reproductive-aged women with polycystic ovary syndrome and association with obesity. Am J Hypertens. (2015) 28:847-51. doi: 10.1093/ajh/hpu251

\section{FUNDING}

This study was supported in part by Taiwan Ministry of Health and Welfare Clinical Trial Center (MOHW109-TDUB-212-114004), MOST Clinical Trial Consortium for Stroke (MOST 108-2321-B-039-003-), Tseng-Lien Lin Foundation, Taichung, and Changhua Christian Hospital (108-CCH-IRP060), Changhua, Taiwan.

\section{ACKNOWLEDGMENTS}

We would like to thank Enago (www.enago.com) for the English language review (CHEWCT-6).

14. Christakou CD, Diamanti-Kandarakis E. Role of androgen excess on metabolic aberrations and cardiovascular risk in women with polycystic ovary syndrome. Womens Health. (2008) 4:583-94. doi: 10.2217/17455057.4.6.583

15. Amiri M, Ramezani Tehrani F, Behboudi-Gandevani S, Bidhendi-Yarandi R, Carmina E. Risk of hypertension in women with polycystic ovary syndrome: a systematic review, meta-analysis and meta-regression. Reprod Biol Endocrinol. (2020) 18:23. doi: 10.1186/s12958-020-00576-1

16. Zhu S, Zhang B, Jiang X, Li Z, Zhao S, Cui L, et al. Metabolic disturbances in non-obese women with polycystic ovary syndrome: a systematic review and meta-analysis. Fertil Steril. (2019) 111:16877. doi: 10.1016/j.fertnstert.2018.09.013

17. Kalaitzidis RG, Elisaf MS. Treatment of hypertension in chronic kidney disease. Curr Hypertens Rep. (2018) 20:64. doi: 10.1007/s11906-018-0864-0

18. Ke C, Zhu X, Zhang Y, Shen Y. Metabolomic characterization of hypertension and dyslipidemia. Metabolomics. (2018) 14:117. doi: 10.1007/s11306-018-1408-y

19. Cryer MJ, Horani T, DiPette DJ. Diabetes and hypertension: a comparative review of current guidelines. J Clin Hypertens. (2016) 18:95-100. doi: 10.1111/jch.12638

20. Zolotareva O, Saik OV, Konigs C, Bragina EY, Goncharova IA, Freidin $\mathrm{MB}$, et al. Comorbidity of asthma and hypertension may be mediated by shared genetic dysregulation and drug side effects. Sci Rep. (2019) 9:16302. doi: 10.1038/s41598-019-52762-w

21. Xue B, Head J, McMunn A. The impact of retirement on cardiovascular disease and its risk factors: a systematic review of longitudinal studies. Gerontologist. (2020) 60:e367-77. doi: 10.1093/geront/gnz062

22. Liu Q, Xie YJ, Qu LH, Zhang MX, Mo ZC. Dyslipidemia involvement in the development of polycystic ovary syndrome. Taiwan J Obstet Gynecol. (2019) 58:447-53. doi: 10.1016/j.tjog.2019.05.003

23. Patil CN, Racusen LC, Reckelhoff JF. Consequences of advanced aging on renal function in chronic hyperandrogenemic female rat model: implications for aging women with polycystic ovary syndrome. Physiol Rep. (2017) 5:e13461. doi: 10.14814/phy2.13461

24. Zierau L, Gade EJ, Lindenberg S, Backer V, Thomsen SF. Coexistence of asthma and polycystic ovary syndrome: a concise review. Respir Med. (2016) 119:155-9. doi: 10.1016/j.rmed.2016.08.025

25. Tang R, Fraser A, Magnus MC. Female reproductive history in relation to chronic obstructive pulmonary disease and lung function in UK biobank: a prospective population-based cohort study. BMJ Open. (2019) 9:e030318. doi: 10.1136/bmjopen-2019-030318

26. Pan ML, Chen LR, Tsao HM, Chen KH. Relationship between polycystic ovarian syndrome and subsequent gestational diabetes mellitus: a nationwide population-based study. PLoS ONE. (2015) 10:e0140544. doi: 10.1371/journal.pone.0140544

27. Harnod T, Chen W, Wang JH, Lin SZ, Ding DC. Association between depression risk and polycystic ovarian syndrome in young women: a retrospective nationwide population-based cohort study (1998-2013). Hum Reprod. (2019) 34:1830-7. doi: 10.1093/humrep/dez081 
28. Marchesan LB, Spritzer PM. ACC/AHA 2017 definition of high blood pressure: implications for women with polycystic ovary syndrome. Fertil Steril. (2019) 111:579-87.e1. doi: 10.1016/j.fertnstert.2018.11.034

29. Macut D, Mladenovic V, Bjekic-Macut J, Livadas S, Stanojlovic O, Hrncic $\mathrm{D}$, et al. Hypertension in polycystic ovary syndrome: novel insights. Curr Hypertens Rev. (2020) 16:55-60. doi: 10.2174/1573402115666190531071422

30. Luque-Ramirez M, Alvarez-Blasco F, Mendieta-Azcona C, Botella-Carretero JI, Escobar-Morreale HF. Obesity is the major determinant of the abnormalities in blood pressure found in young women with the polycystic ovary syndrome. J Clin Endocrinol Metab. (2007) 92:21418. doi: 10.1210/jc.2007-0190

31. Mioni R, Ca AD, Turra J, Azzolini S, Xamin N, Bleve L, et al. Hyperinsulinemia and obese phenotype differently influence blood pressure in young normotensive patients with polycystic ovary syndrome. Endocrine. (2017) 55:625-34. doi: 10.1007/s12020-016-0958-x

32. Di Domenico K, Wiltgen D, Nickel FJ, Magalhaes JA, Moraes RS, Spritzer PM. Cardiac autonomic modulation in polycystic ovary syndrome: does the phenotype matter? Fertil Steril. (2013) 99:286-92. doi: 10.1016/j.fertnstert.2012.08.049

33. Diamanti-Kandarakis E, Dunaif A. Insulin resistance and the polycystic ovary syndrome revisited: an update on mechanisms and implications. Endocr Rev. (2012) 33:981-1030. doi: 10.1210/er.2011-1034

34. Yanes Cardozo LL, Romero DG, Reckelhoff JF. Cardiometabolic features of polycystic ovary syndrome: role of androgens. Physiology. (2017) 32:35766. doi: 10.1152/physiol.00030.2016

35. Wild RA, Carmina E, Diamanti-Kandarakis E, Dokras A, Escobar-Morreale HF, Futterweit W, et al. Assessment of cardiovascular risk and prevention of cardiovascular disease in women with the polycystic ovary syndrome: a consensus statement by the androgen excess and polycystic ovary syndrome (AE-PCOS) society. J Clin Endocrinol Metab. (2010) 95:203849. doi: $10.1210 /$ jc. $2009-2724$

36. Gunning MN, Fauser B. Are women with polycystic ovary syndrome at increased cardiovascular disease risk later in life? Climacteric. (2017) 20:2227. doi: 10.1080/13697137.2017.1316256

37. Azziz R. Polycystic ovary syndrome. Obstet Gynecol. (2018) 132:32136. doi: 10.1097/AOG.0000000000002698

38. Teede HJ, Misso ML, Costello MF, Dokras A, Laven J, Moran L, et al. Recommendations from the international evidence-based guideline for the assessment and management of polycystic ovary syndrome. Hum Reprod. (2018) 33:1602-18. doi: 10.1093/humrep/dey256

39. Li C, Binongo JN, Kancherla V. Effect of parity on pregnancy-associated hypertension among Asian American women in the United States. Matern Child Health J. (2019) 23:1098-107. doi: 10.1007/s10995-01902746-Z

Conflict of Interest: The authors declare that the research was conducted in the absence of any commercial or financial relationships that could be construed as a potential conflict of interest.

Copyright (c) $2020 \mathrm{Wu}$, Chiu, Chang, Lee, Lee, Lee and Wei. This is an open-access article distributed under the terms of the Creative Commons Attribution License (CC $B Y)$. The use, distribution or reproduction in other forums is permitted, provided the original author(s) and the copyright owner(s) are credited and that the original publication in this journal is cited, in accordance with accepted academic practice. No use, distribution or reproduction is permitted which does not comply with these terms. 